International Institute for Applied Systems Analysis • A-2361 Laxenburg • Austria Tel: +432236807 • Fax: +43223671313• E-mail: info@iiasa.ac.at•Web: www.iiasa.ac.at

INTERIM REPORT IR-97-042/ November

\title{
Food Chains in the Chemostat: Relationships Between Mean Yield and Complex Dynamics
}

Alessandra Gragnani (gragnani@elet.polimi.it)

OscarDeFeo (odefeo@circhp.epfl.ch)

Sergio Rinaldi (rinaldi@elet.polimi.it)

\author{
Approved by \\ Ulf Dieckmann (dieckman@iiasa.ac.at) \\ Project Leader, Adaptive Dynamics Network
}

Interim Reports on work of the International Institute for Applied Systems Analysis receive only limited review. Views or opinions expressed herein do not necessarily represent those of the Institute, its National Member Organizations, or other organizations supporting the work. 


\section{Contents}

1 Introduction $\quad 1$

2 The models $\quad 4$

$\begin{array}{lll}3 & \text { Bifurcation analysis and complex dynamics } & 6\end{array}$

4 Mean yield 10

5 Conclusion $\quad 11$ 


\title{
About the Authors
}

\author{
Alessandra Gragnani \\ Politecnico di Milano \\ I-20133 Milano, Italy \\ Oscar De Feo \\ Swiss Federal Institute of Technology \\ CH-1015 Lausanne, Switzerland \\ and \\ Adaptive Dynamics Network \\ International Institute for Applied Systems Analysis \\ A-2361 Laxenburg, Austria \\ Sergio Rinaldi \\ Dipartimento di Elettronica e Informazione ${ }^{1}$ \\ Politecnico di Milano \\ I-20133 Milano, Italy \\ Via Ponzio 34/5 \\ 20133 Milano, Italy \\ phone: $+39-2-23993563$ \\ fax: $+39-2-23993412$ \\ e-mail: rinaldi@elet.polimi.it \\ and \\ Adaptive Dynamics Network \\ International Institute for Applied Systems Analysis \\ A-2361 Laxenburg, Austria.
}

\section{Acknowledgements}

This article was written at the International Institute for Applied Systems Analysis (IIASA), Laxenburg, Austria, where the authors cooperated with the Adaptive Dynamics Network project. The work has been financially supported by the Italian Ministry of Scientific Research and Technology (MURST), contract Teoria dei sistemi e del controllo.

\footnotetext{
${ }^{1}$ Address for further correspondence
} 


\begin{abstract}
A tritrophic food chain chemostat model composed of a prey with Monod type nutrient uptake, a Holling type II predator and a Holling type II exploited superpredator is considered in this paper. The bifurcations of the model show that dynamic complexity first increases and then decreases with the nutrient supplied to the bottom of the food chain. Extensive simulations prove that the same holds for food yield, i.e., there exists an optimum nutrient supply which maximizes mean food yield. Finally, a comparative analysis of the results points out that the optimum nutrient supply practically coincides with the nutrient supply separating chaotic dynamics from high-frequency cyclic dynamics. This reinforces the idea, already known for simpler models, that food yield maximization requires that the system behaves on the edge of chaos.
\end{abstract}




\title{
Food Chains in the Chemostat: Relationships Between Mean Yield and Complex Dynamics
}

\author{
Alessandra Gragnani \\ Oscar De Feo \\ Sergio Rinaldi
}

\section{Introduction}

Aim of this paper is to investigate, through bifurcation analysis (Guckenheimer and Holmes, 1983), the relationships between dynamic complexity and mean yield of exploited food chains. This topic was first investigated by Rosenzweig in his famous paper on the paradox of enrichment (Rosenzweig, 1971), where he says "Man must be very careful in attempting to enrich an ecosystem in order to increase its food yield. There is a real chance that such activity may result in decimation of the food species that are wanted in greater abundance". Rosenzweig's analysis is based on a ditrophic food chain model composed of a logistic prey $x_{1}$ and a predator $x_{2}$ with saturating functional response. Enrichment is performed by increasing the prey carrying capacity $K$ while harvest is exerted at constant effort so that mean food yield is proportional to mean predator abundance $\bar{x}_{2}$.

Indeed, it has been shown (May, 1972; Gilpin, 1972) that for low prey carrying capacities such food chains settle to a positive equilibrium, while for higher carrying capacities the asymptotic regime is cyclic. The critical value $K^{*}$ of the carrying capacity separating stationary from cyclic regimes corresponds to a supercritical Hopf bifurcation (Sarkar et al., 1991). In other words, the positive equilibrium loses stability when the carrying capacity is increased and when it becomes unstable, the attractor becomes a stable limit cycle. Moreover, it is easy to derive analytically that for $K<K^{*}$ predator biomass at equilibrium increases with carrying capacity. Finally, it can be proved (De Feo and Rinaldi, 1997) that if prey time response is remarkably smaller than predator time response, the mean value of predator biomass for $K>K^{*}$ is lower than the equilibrium value for $K=K^{*}$. Thus, in conclusion, the mean yield associated to the attractor of a ditrophic food chain first increases and then decreases with prey carrying capacity and reaches its maximum for $K=K^{*}$, i.e., when the dynamic behavior becomes cyclic. The above results suggest the use of the following simple operating rules if one is interested in maximizing mean yield

- if a ditrophic food chain is stationary, then increase nutrient supply

- if a ditrophic food chain is cyclic, then decrease nutrient supply 
The systematic use of these rules should slowly push exploited ditrophic food chains to behave on the edge of their most complex dynamic regime, namely the cyclic regime.

Recently, the study of the relationships between dynamic complexity and mean yield has been extended to tritrophic food chains composed of a logistic prey $x_{1}$, a Holling type II predator $x_{2}$ and a Holling type II exploited superpredator $x_{3}$, i.e., the so-called Rosenzweig-MacArthur tritrophic food chain. Such food chains have a very rich behavior, covering the whole spectrum of dynamic regimes, including chaos (Hogeweg and Hesper, 1978; Scheffer, 1991; Hastings and Powell, 1991; McCann and Yodzis, 1994; Abrams and Roth, 1994). Detailed bifurcation analysis performed by different authors (Klebanoff and Hastings, 1994; McCann and Yodzis, 1995; Kuznetsov and Rinaldi, 1996) show that stationary, cyclic and chaotic coexistence is possible. Moreover, food chains with time responses increasing from bottom to top have cyclic regimes which are either low-frequency or high-frequency regimes (Muratori and Rinaldi, 1992). The geometry of the corresponding limit cycles is shown in Fig. 1. The low-frequency limit cycles (Fig. 1a) are characterized by large and slow variations of the superpredator and by high-frequency bursts of the prey and predator communities. By contrast, high-frequency limit cycles (Fig. 1b) are characterized by almost steady superpredator populations. In conclusion, Rosenzweig-MacArthur food chains can be grouped into five classes (those for which coexistence is not possible and those for which coexistence is stationary, cyclic at low-frequency, cyclic at high-frequency, and chaotic) and the boundaries of such classes in parameter space can be explicitly produced through numerical bifurcation analysis. In general, the region of chaotic coexistence in any two-dimensional parameter space is a relatively narrow fractal band delimited on one side by the region of low-frequency cyclic coexistence and on the other side by the region of high-frequency cyclic coexistence (Kuznetsov and Rinaldi, 1996). Consistently, on one side the strange attractor resembles the low-frequency limit cycle shown in Fig. 1a and is called "teacup" attractor (Hastings and Powell, 1991), while on the other side the strange attractor looks like the high-frequency limit cycle shown in Fig. 1b and is called "cut teacup" attractor.

The influence of prey carrying capacity on superpredator mean abundance $\bar{x}_{3}$ has also been investigated through simulation (Abrams and Roth, 1994a,b) and the results suggest that in many circumstances $\bar{x}_{3}$ first increases and then decreases with prey carrying capacity. It is therefore possible to simply classify food chains in under- and over-supplied food chains (De Feo and Rinaldi, 1997). The first ones are those in which a small increase of prey carrying capacity gives rise to a small increase of mean abundance of the top trophic level. Viceversa, over-supplied food chains are those that can be marginally improved through impoverishment.

Although, in principle, one should not expect any particular relationship between the two above classifications of food chains, it has been recently shown (De Feo and Rinaldi, 1997), that a very strong relationship exists, namely high-frequency cyclic food chains are over-supplied, while all other food chains are under-supplied. The most intriguing implication of this discovery is that food chains with maximum mean yield are on the edge of their most complex behavior, namely a chaotic regime. Obviously, this conclusion generalizes the above described findings on ditrophic food 


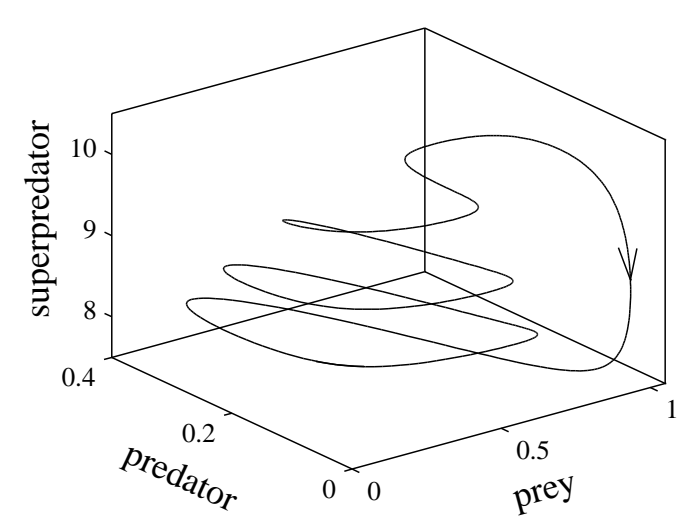

(a)

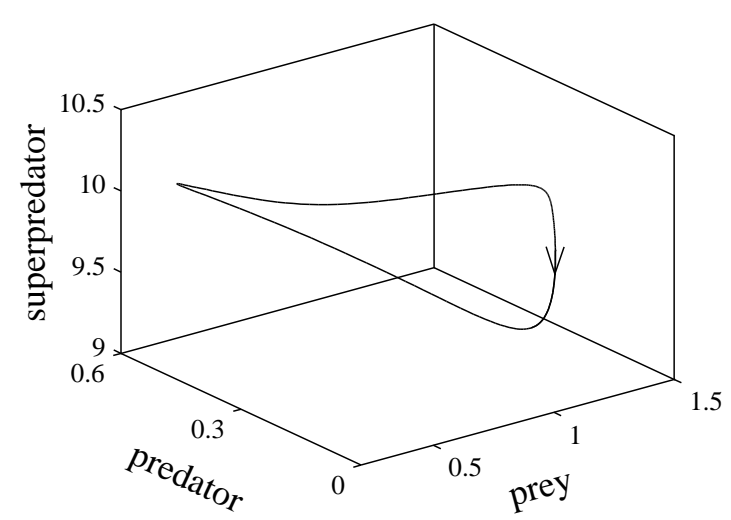

(b)

Figure 1: Low-frequency (a) and high-frequency (b) limit cycles of RosenzweigMacArthur tritrophic food chain $\left(x_{1}=\right.$ prey, $x_{2}=$ predator, $x_{3}=$ superpredator $)$.

chains. Consistently, one can generalize the two operating rules mentioned above in the following way:

(i) if a tritrophic food chain is stationary, cyclic at low-frequency or chaotic, then increase nutrient supply

(ii) if a tritrophic food chain is cyclic at high-frequency, then decrease nutrient supply

It is important to notice that these rules allow one to take a decision about enriching or impoverishing an exploited food chain even in the absence of informations on system parameters. Moreover, the systematic application of these rules should slowly improve mean food yield and gradually transform food chains with simple behavior into chaotic or almost chaotic food chains.

Since all the above results have been derived by analyzing food chain models in which the prey is logistic, it is of interest to determine if such results hold also in the case the prey feeds on a limiting nutrient $x_{0}$ available in a pool. For this reason, the Rosenzweig-MacArthur tritrophic food chain is complemented by an extra differential equation for the nutrient. Such an equation is simply the balance of the various flows regulating the nutrient concentration, namely inflow and outflow rates, nutrient recycling due to decomposition of dead individuals of the three populations and nutrient uptake rate of the prey population. Assuming that the nutrient uptake per unit of prey is a Monod function of the nutrient, the model turns out to be a natural extension of the prey-predator chemostat model proposed by Canale $(1969,1970)$ and later used in many studies of protozoan predation on bacteria and of aquatic ecosystems (e.g., Canale et al., 1973; Jost et al., 1973; Drake and Tsuchiya, 1976; Bader et al., 1976). Such a ditrophic food chain model has been deeply investigated (Butler et al., 1983; Cunningham and Nisbet, 1983; Waltman, 1983) and the result is that for sufficiently low inflow and outflow rates the dynamics observed in the chemostat are the same as those predicted by the Rosenzweig-MacArthur ditrophic food chain, i.e., for any parameter setting there is only one attractor which is either 
an equilibrium or a limit cycle. By contrast, for too high inflow and outflow rates prey and, hence, predator cannot survive because they are simply washed out from the chemostat. The dynamics of Canale's chemostat model have been also studied in the case of periodically varying parameters (Kot et al., 1992; Pavlou and Kevrekidis, 1992) and shown to be equivalent (except for washout) to those of the periodically forced Rosenzweig-MacArthur model (Kuznetsov et al., 1992; Rinaldi et al., 1993; Gragnani and Rinaldi, 1995).

Aim of this paper is to study the dynamics of tritrophic food chains embedded in the chemostat and to show, once more, that such dynamics are equivalent (except for washout phenomena) to those of the Rosenzweig-MacArthur tritrophic food chain. This will technically be accomplished by comparing the bifurcation diagrams of the two models and, in particular, their codimension-2 bifurcation points. Moreover, it will be shown that the relationship between dynamic complexity and mean yield pointed out for Rosenzweig-MacArthur food chains holds also in the case of a tritrophic food chain embedded in a chemostat. This is the most important contribution of the paper. Theoretically, it shows that the equivalence between the two models is really profound; practically it suggests interesting operating rules for the management of exploited aquatic ecosystems.

\section{The models}

The Rosenzweig-MacArthur model of tritrophic food chains is the following

$$
\begin{aligned}
\frac{d x_{1}}{d t} & =x_{1}\left[r\left(1-\frac{x_{1}}{K}\right)-\frac{a_{2} x_{2}}{b_{2}+x_{1}}\right] \\
\frac{d x_{2}}{d t} & =x_{2}\left[e_{2} \frac{a_{2} x_{1}}{b_{2}+x_{1}}-\frac{a_{3} x_{3}}{b_{3}+x_{2}}-d_{2}\right] \\
\frac{d x_{3}}{d t} & =x_{3}\left[e_{3} \frac{a_{3} x_{2}}{b_{3}+x_{2}}-d_{3}\right]
\end{aligned}
$$

where $x_{1}, x_{2}$, and $x_{3}$ are prey, predator and superpredator biomass, $r$ and $K$ are prey growth rate and carrying capacity, and $a_{i}, b_{i}, e_{i}$, and $d_{i}, i=2,3$, are maximum predation rate, half saturation constant, efficiency, and death rate of predator $(i=2)$ and superpredator $(i=3)$. If the superpredator is exploited, its mortality $d_{3}$ is the sum of basic mortality and harvesting effort, so that food yield is proportional to superpredator biomass. Given a food chain, it is always possible, at least in principle, to enrich it or impoverish it by increasing or decreasing the supply of nutrients to the bottom of the chain. This can be realized through various actions which very often influence only the prey carrying capacity $K$ (Oksanen et al., 1981; Abrams, 1993) but can also influence the prey growth rate (De Feo and Rinaldi, 1997).

As already said, tritrophic food chains embedded in a chemostat can be described by adding to the variables $x_{1}, x_{2}$, and $x_{3}$ an extra state variable $x_{0}$ denoting the concentration of the nutrient in the chemostat. The corresponding model, from now on called Canale's model, is the following

$$
\frac{d x_{0}}{d t}=D\left(x_{i}-x_{0}\right)-\frac{a_{1} x_{0}}{b_{1}+x_{0}} x_{1}
$$




$$
\begin{aligned}
\frac{d x_{1}}{d t} & =x_{1}\left[e_{1} \frac{a_{1} x_{0}}{b_{1}+x_{0}}-\frac{a_{2} x_{2}}{b_{2}+x_{1}}-d_{1}-\epsilon_{1} D\right] \\
\frac{d x_{2}}{d t} & =x_{2}\left[e_{2} \frac{a_{2} x_{1}}{b_{2}+x_{1}}-\frac{a_{3} x_{3}}{b_{3}+x_{2}}-d_{2}-\epsilon_{2} D\right] \\
\frac{d x_{3}}{d t} & =x_{3}\left[e_{3} \frac{a_{3} x_{2}}{b_{3}+x_{2}}-d_{3}-\epsilon_{3} D\right]
\end{aligned}
$$

where $D$ is the constant inflow rate (equal to outflow rate), $x_{i}$ is the constant concentration of the nutrient in the inflow, $a_{1} x_{0} /\left(b_{1}+x_{0}\right)$ is the nutrient uptake per unit prey, and $\epsilon_{i} D, i=1,2,3$, is the washout rate of the $i$-th population. If the superpredator is exploited, its mortality rate $d_{3}$ comprises also the harvesting effort and again food yield is proportional to superpredator biomass. Moreover, enrichment can be performed by increasing the inflow rate $D$ and/or the nutrient concentration $x_{i}$ of the inflow.

The parameters controlling the enrichment, namely $(K, r)$ for model (1) and $\left(x_{i}, D\right)$ for model $(2)$, are the parameters with respect to which bifurcation analysis will be performed. All other parameters will be kept fixed at specified reference values. For model (1) these are: $a_{2}=5 / 3, b_{2}=1 / 3, e_{2}=1, d_{2}=0.4, a_{3}=$ $0.05, b_{3}=0.5, e_{3}=1, d_{3}=0.01$, while for model $(2)$ they are $a_{1}=1.25, b_{1}=$ $8, e_{1}=0.4, d_{1}=0.01, \epsilon_{1}=1, a_{2}=0.33, b_{2}=9, e_{2}=0.6, d_{2}=0.001, \epsilon_{2}=$ $0.8, a_{3}=0.021, \quad b_{3}=15.19, e_{3}=0.9, d_{3}=0.0001, \epsilon_{3}=0.1$. The reference parameter values selected for model (1) have already been used by many authors (Hastings and Powell, 1991; Klebanoff and Hastings, 1994; Abrams and Roth, 1994a; Kuznetsov and Rinaldi, 1996; De Feo and Rinaldi, 1997, 1998). The reference parameter values selected for model (2) are a complement of those used by some authors for ditrophic food chains (Cunningham and Nisbet, 1983; Kot et al., 1992) and are not too different from those used by Kooi et al. (1997) and Boer et al. (1997) for tritrophic food chains. Notice that in both parameter settings death and predation rates are decreasing with the trophic level, indicating that the time responses increase from bottom to top.

It is important to notice that the chemostat models considered in the literature are very often a special case of model (2), namely that corresponding to

$$
\epsilon_{i}=1, \quad d_{i}=0 \quad i=1,2,3
$$

Under these circumstances it is straightforward to verify that the variable

$$
X(t)=x_{0}(t)+\frac{x_{1}(t)}{e_{1}}+\frac{x_{2}(t)}{e_{1} e_{2}}+\frac{x_{3}(t)}{e_{1} e_{2} e_{3}}-x_{i}
$$

obeys the differential equation

$$
\frac{d X(t)}{d t}=-D X(t)
$$

and therefore tends asymptotically to zero. This implies that the asymptotic modes of behavior of such a chemostat can be studied by eliminating one state variable, say the nutrient $x_{0}(t)$, which can be computed for large values of $t$ from the other state variables with the formula

$$
x_{0}(t)=x_{i}-\frac{x_{1}(t)}{e_{1}}-\frac{x_{2}(t)}{e_{1} e_{2}}-\frac{x_{3}(t)}{e_{1} e_{2} e_{3}} .
$$


In conclusion, under assumptions (3) the asymptotic behavior of the fourth order chemostat model (2) can be analyzed by studying a reduced third order chemostat model. One could therefore naturally expect that such a reduced model has strong similarities with the Rosenzweig-MacArthur model (1) which is also a third order model. Nevertheless, conditions (3) are questionable at least for three reasons. First, because in many food chains (for example, phytoplankton - zooplankton - fish) the individuals of the highest trophic levels have the capability of resisting to washout, so that $\epsilon_{i}<1$, at least for $i=3$. Second, because the assumption $d_{i}=0$, supported by the observation that basic death rate is negligeable with respect to washout rate $D$, would limit the use of model (2) to aquatic ecosystems with relatively high inflow and outflow rates. Third, because condition $d_{3}=0$ is in conflict with the assumption that the food chain is exploited by harvesting the superpredator. For all these reasons, the reference parameter values of the chemostat model do not satisfy conditions (3) and the analysis is performed directly on model (2) and not on a reduced order model. Thus, one cannot argue that the strong similarities that will be shown to exist between models (1) and (2) could be simply discovered through a suitable transformation like eq. (4).

\section{Bifurcation analysis and complex dynamics}

The bifurcations of models (1) and (2) must be computed in order to make a sound comparative analysis. The study is performed by varying two control parameters, namely $K$ and $r$ for model (1) and $x_{i}$ and $D$ for model (2). The choice of these control parameters is dictated by the aim of the study which focuses on the role of enrichment. All other parameters are kept constant at the reference values mentioned in the previous section. The analysis of the equilibria of models (1) and (2) and of their local bifurcations has been carried out analytically or by means of specialized software for algebraic manipulation. By contrast, the analysis of the limit cycles and their local bifurcations has been performed numerically by means of AUTO (Doedel and Kenévez, 1986). Finally, the analysis of global bifurcations (mainly homoclinic bifurcations) has been carried out by means of a continuation method described in Champneys and Kuznetsov (1994). Details on the methodology are not reported here because they are already available in the literature (Kuznetsov and Rinaldi, 1996; De Feo and Rinaldi, 1997).

The results of the analysis are shown in Fig. 2, where two bifurcation diagrams are reported, the first concerning model (1) and the second model (2).

The symbols identifying each bifurcation curve have the following meaning:

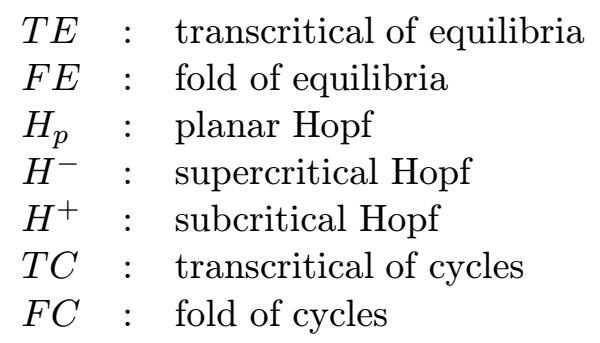

The continuous lines refer to catastrophic bifurcations of attractors, and the dashed ones refer to non-catastrophic bifurcations of attractors or to bifurcations of saddles 


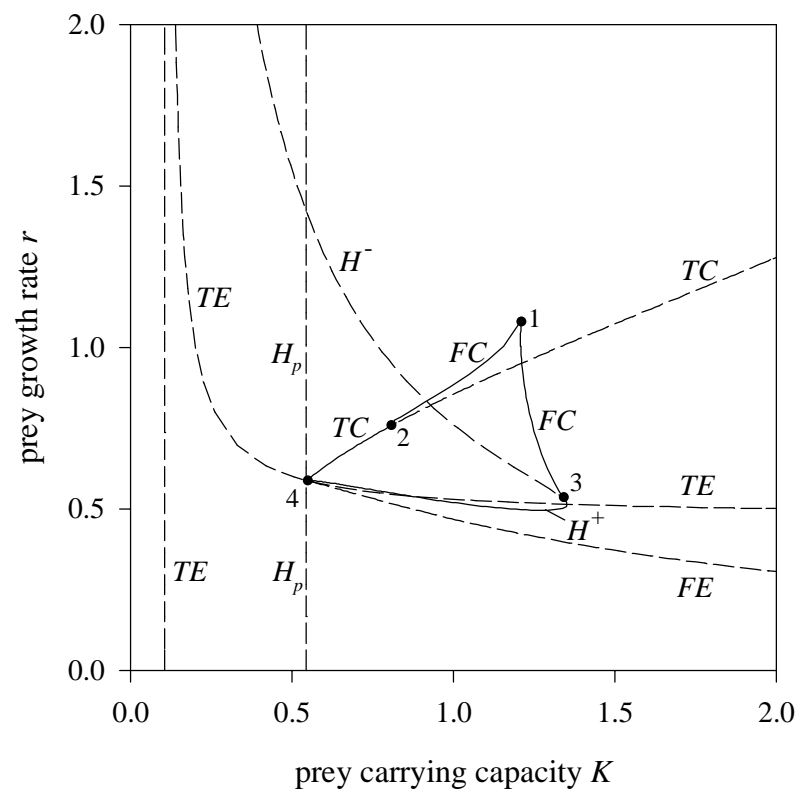

(a)

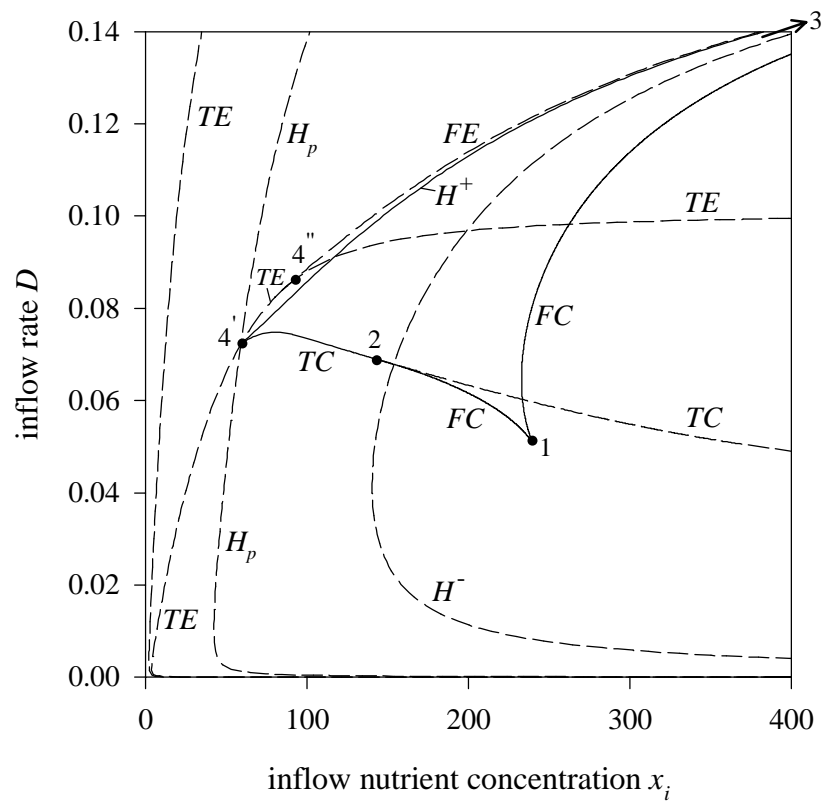

(b)

Figure 2: Bifurcation diagrams of Rosenzweig-MacArthur model (a) and Canale's model (b). Continuous curves refer to catastrophic bifurcations. Dashed curves are bifurcations of saddles and repellors or non-catastrophic bifurcations of attractors. For parameter values and label interpretations see text. 
and repellors. When a continuous line is crossed, the attractor changes macroscopically. By contrast, when a dashed line is crossed, there is no discontinuity in the attractor and the dynamic regime changes smoothly.

The two diagrams are not complete since some curves produced with AUTO (in particular, flip and fold bifurcation curves forming the boundary of the region of chaotic coexistence) have not been drawn for simplicity. Readers interested in more details on the Rosenzweig-MacArthur model can refer to Kuznetsov and Rinaldi (1996) for the bifurcations delimiting the region of chaotic coexistence and to De Feo and Rinaldi (1998) for some results on homoclinic bifurcations, while readers interested in Canale's model can refer to Kooi et al. (1997) and Boer et al. (1997) where a bifurcation diagram similar to that of Fig. $2 \mathrm{~b}$ is presented for a different parameter setting and discussed with emphasis on global bifurcations.

In order to recognize that the two bifurcation diagrams of Fig. 2 are similar one to each other, one should concentrate on their codimension- 2 bifurcation points, which are the points where two or more bifurcation curves merge (Kuznetsov, 1995). These points are sometimes called organizing centers, because they determine the structure of the entire bifurcation diagram.

In Fig. 2a, there are four organizing centers (see points 1,2,3,4), while in Fig. $2 \mathrm{~b}$ the organizing centers are five (see points $1,2,3,4^{\prime}, 4^{\prime \prime}$ ). In both diagrams point 1 is a cusp where two fold bifurcation curves of limit cycles $F C$ merge. Thus point 1 is the same codimension- 2 bifurcation point for the two models. The reader can easily check that the same is true for points 2 and 3. By contrast, point 4 in Fig. 2a is split into two points, namely $4^{\prime}$ and $4^{\prime \prime}$, in Fig. $2 \mathrm{~b}$, since the bifurcation curves merging in point 4 are exactly those merging in points $4^{\prime}$ and $4^{\prime \prime}$. Thus, in conclusion, the structure of the two bifurcation diagrams is the same.

The similarity of the two models can be more clearly verified by extracting from Fig. 2 the regions of stationary, cyclic and chaotic coexistence of the three populations. This can be done by looking only at the bifurcations of the most significant attractor, namely that which has the highest mean yield. In other words, all bifurcation curves of Fig. 2 dealing with saddles, repellors or other attractors that coexist with the main one in some small regions of parameter space have been disregarded, thus producing the two diagrams of Fig. 3. Such a figure gives a good idea of the influence of the two control parameters on the dynamics of the two food chain models. When $r$ is low in the Rosenzweig-MacArthur model and when $D$ is high in Canale's model, the superpredator goes extinct so that food yield is zero. In the remaining regions, going from the left to the right, i.e., enriching, one finds stationary coexistence, cyclic coexistence at low frequency, chaotic coexistence and, finally, cyclic coexistence at high-frequency. The chaotic region is a rather narrow band delimited by a regular curve on the right side and by a fractal set produced by a very complex bifurcation structure on the left side. Strange attractors close to this border are teacup strange attractors similar to the limit cycle shown in Fig. 1a. By contrast, strange attractors close to the opposite border are cut teacup strange attractors and resemble the high-frequency limit cycle shown in Fig. 1b. This is evident in Fig. 4 where the attractors corresponding to points $A, B, C, D$ of Fig. $3 \mathrm{~b}$ are shown.

In conclusion, one can say that both tritrophic food chain models produce the 


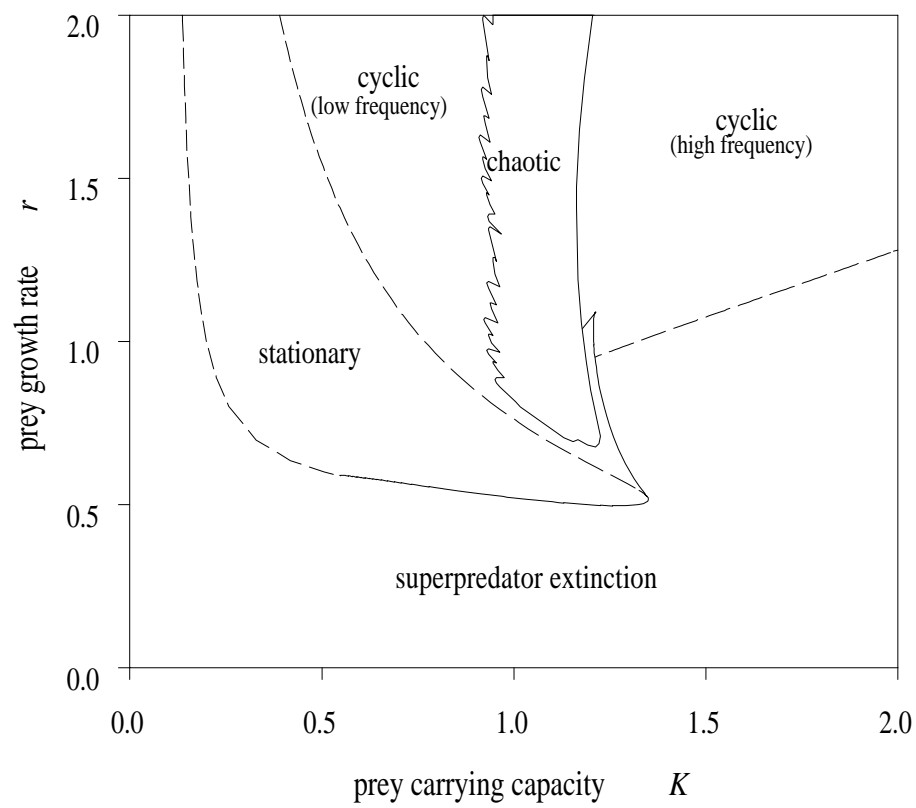

(a)

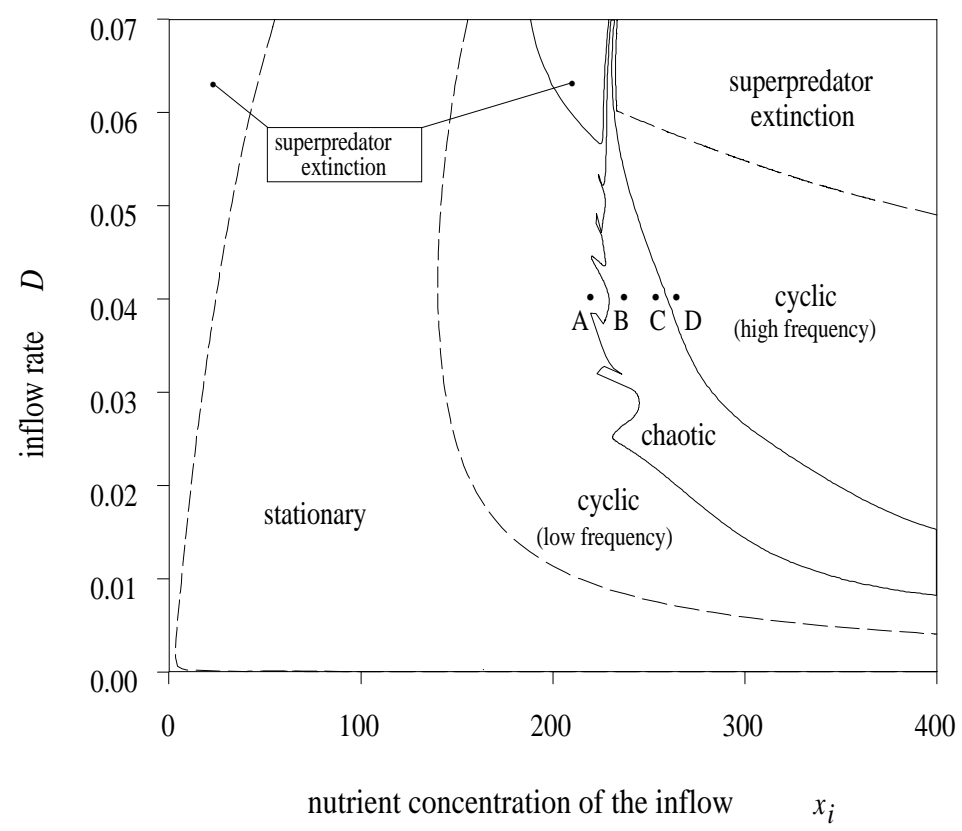

(b)

Figure 3: Regions of prey, predator and superpredator coexistence characterized by different dynamic regimes: (a) Rosenzweig-MacArthur model, (b) Canale's model (points $A, B, C, D$ refer to next figure). 


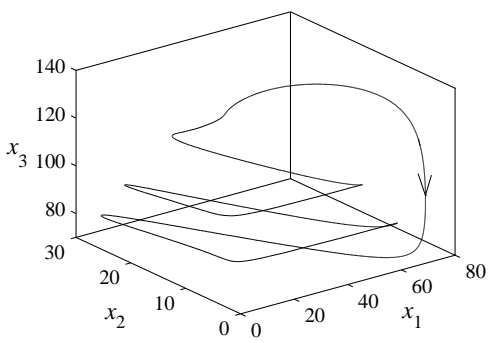

(a)

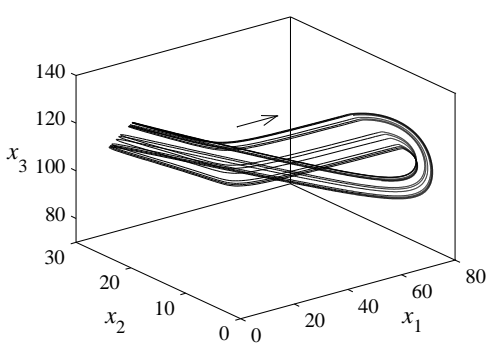

(c)

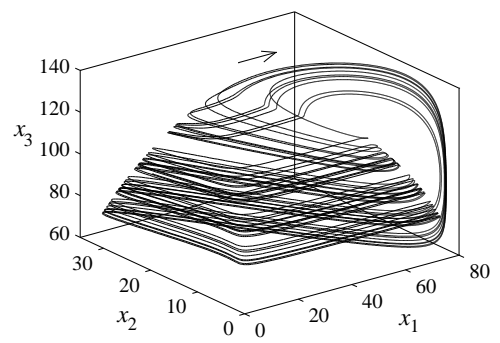

(b)

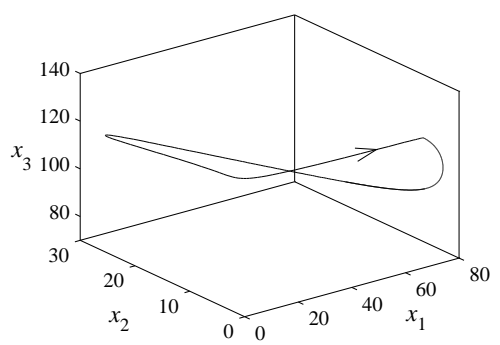

(d)

Figure 4: Four attractors of Canale's model: (a) low-frequency limit cycle; (b) teacup strange attractor; (c) cut teacup strange attractor; (d) high-frequency limit cycle. The attractors correspond to the parameters values $\left(x_{i}, D\right)$ indicated in Fig. 3b with points $A, B, C, D$.

same dynamics and the same scenarios when a parameter is varied. In particular, for a continuous enrichment of the food chain, the complexity of the dynamic behaviour first increases, from stationary to cyclic and, finally, to chaotic coexistence and then decreases, from chaotic to cyclic coexistence. Thus, the region of chaotic coexistence is embedded in the region of cyclic coexistence and such a cyclic coexistence is at low-frequency on one side and at high-frequency on the other.

\section{Mean yield}

This section is fully devoted to Canale's model and, in particular, to mean food yield and its dependence on flow rate $D$ and concentration $x_{i}$ of the inflow of the chemostat. As already said, the mean superpredator abundance $\bar{x}_{3}$ will be considered as an indicator of food yield. Since the function $\bar{x}_{3}\left(x_{i}, D\right)$ cannot be determined analytically, the value of $\bar{x}_{3}$ has been computed numerically on a fine grid of about 10,000 points in the control space $\left(x_{i}, D\right)$. A special program based on spectral analysis was used to compute $\bar{x}_{3}$ when the regime of coexistence was chaotic. Finally, the results of these computations were interpolated to produce the graph of the function $\bar{x}_{3}\left(x_{i}, D\right)$ shown in Fig. 5. Obviously, food yield is zero in the regions of superpredator extinction (see Fig. 3b), while it is first increasing and then decreasing with the concentration of the inflow $x_{i}$, meaning that there is an optimum nutrient supply that maximizes mean food yield.

But this is not the most interesting result. Indeed, sharper conclusions can be derived by carefully analyzing the figures obtained so far. In fact, if the crest of 


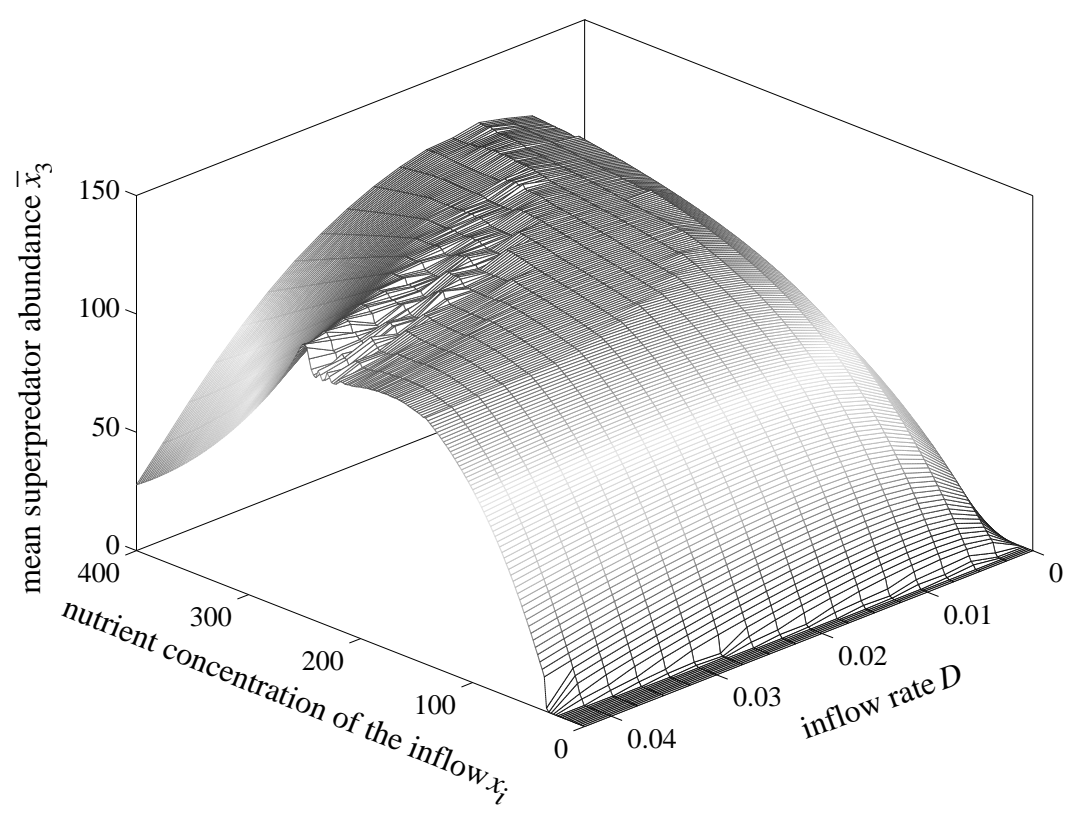

Figure 5: The graph of the function $\left(\bar{x}_{3}\left(x_{i}, D\right)\right.$ describing the dependence of mean superpredator abundance $\bar{x}_{3}$ upon concentration $x_{i}$ and flow rate $D$ of nutrient inflow.

the surface of Fig. 5 (i.e., the line on which $\bar{x}_{3}$ is maximum with respect to $x_{i}$ ) is projected on the plane $\left(x_{i}, D\right)$, the sets of under- and over-supplied food chains in the control space are obtained. Then, if such sets are superimposed to Fig. 3b, a surprisingly simple result, illustrated in Fig. 6, emerges: stationary, cyclic at low-frequency and chaotic food chains are under-supplied, while food chains cycling at high-frequency are over-supplied. This is exactly the same property holding in Rosenzweig-MacArthur tritrophic food chains (De Feo and Rinaldi, 1997). Thus, once more, the conclusion is that the two tritrophic food chain models (1) and (2) are qualitatively equivalent.

The above property supports the two simple operating rules mentioned in the introduction. These rules could be used, even empirically, to guide any adjustment process aimed at improving food yield and, in principle, their systematic application should slowly push the food chain to behave on the edge of chaos.

\section{Conclusion}

The dynamics of two tritrophic food chain models (Rosenzweig-MacArthur model and Canale's model) have been studied through bifurcation analysis with the aim of proving that the two models are qualitatively similar. The results show, in particular, that dynamic complexity first increases and then decreases with enrichment. More precisely, if the nutrient supplied to the bottom of the food chain is increased, the attractor is first an equilibrium, then a low-frequency limit cycle and finally a chaotic attractor, but if nutrient is further increased, the chaotic attractor becomes a high-frequency limit cycle. 


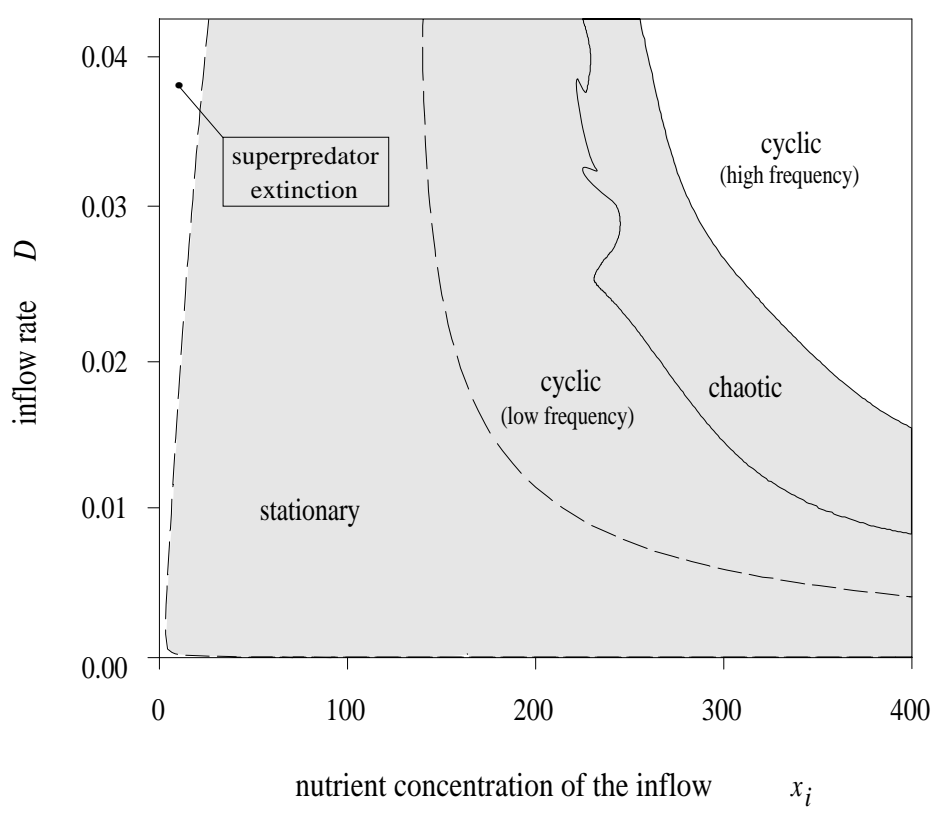

Figure 6: The region of under-supplied food chains (in gray) and the regions of coexistence (see Fig. 3).

Then, the dependence of the mean abundance of the superpredator upon the nutrient supplied to the bottom of the food chain has been established through systematic and detailed simulations. The results show that in both models mean abundance of the superpredator first increases and then decreases with enrichment. This means that in exploited food chains, where the mean food production is proportional to the mean abundance of the superpredator, there exists an optimum nutrient supply. Finally, it has been verified that the optimum nutrient supply practically coincides with the nutrient supply separating chaotic dynamics from high-frequency cyclic dynamics.

All these results imply that one should enrich food chains which are stationary, cyclic at low-frequency or chaotic and impoverish food chains which are cyclic at high-frequency, if the aim is the maximization of mean food yield. If these operating rules were systematically applied, the food chain would sooner or later behave on the edge of chaos. This is in line with the general principle "optimality implies chaos" which has been advocated in other areas of biology, like brain activity (Rapp et al., 1985), heart beating (West and Goldbeter, 1987), metapopulations (Allen et al., 1993), and evolution (Ferrière and Gatto, 1993; Kauffman, 1993).

It is fair to say that the enthusiasm for the principle "optimality implies chaos" should be counterbalanced by the consciousness that the principle holds only under specific assumptions. For example, in the case of tritrophic food chains it can be verified, by repeating for different parameter settings the analysis presented in this paper, that the principle holds when the superpredator has slower dynamics than the two other populations. In the case of extremely diversified dynamics the principle can actually be formally proved through the methods of singular perturbation theory. The proof is not reported here because already available in the paper devoted to Rosenzweig-MacArthur food chains (De Feo and Rinaldi, 1997). 
The results obtained so far for tritrophic food chains are definitely stimulating in view of their potential applications. Along this line, it would be interesting to know if more realistic ecosystems, like the plankton-fish food chain, satisfy the principle "optimality implies chaos", because this might have relevant consequences in the management of natural aquatic ecosystems as well as in aquaculture. Moreover, the same type of analysis carried out here for superpredator mean abundance, could be repeated for other indicators, in order to detect, for example, if there are strict relationships also between primary productivity and dynamic complexity. All these problems deserve further attention.

\section{References}

Abrams, P. A. 1993. Effect of increased productivity on the abundances of trophic levels. The American Naturalist 141, 351-371.

Abrams, P. A. and J. D. Roth. 1994a. The effects of enrichment of three-species food chains with non-linear functional responses. Ecology 75, 1118-1130.

Abrams, P. A. and J. D. Roth. 1994b. The responses of unstable food chains to enrichment. Evolutionary Ecology 8, 150-171.

Allen, J.C., W.M. Schaffer and D. Rosko. 1993. Chaos reduces species extinction by amplifying local population noise. Nature 364, 229-232.

Bader, F. G., H. M. Tsuchiya and A. G. Fredrickson. 1976. Grazing of ciliates on blue-green algae: Effects of ciliate encystment and related phenomena. Biotechnology and Bioengineering 18, 311-332.

Boer, M. P., B. W. Kooi and S.A Kooijman. 1997. Food chain dynamics in the chemostat. Submitted.

Butler, G. J., S. B. Hsu and P. Waltman. 1983. Coexistence of competing predators in a chemostat. Journal of Mathematical Biology 17, 133-151.

Canale, R. P. 1969. Predator-prey relationships in a model for the activated process. Biotechnology and Bioengineering 11, 887-907.

Canale, R. P. 1970. An analysis of models describing predator-prey interaction. Biotechnology and Bioengineering 12, 353-378.

Canale, R. P., T. D. Lustig, P. M. Kehrberger and J. E. Salo. 1973. Experimental and mathematical modeling studies of protozoan predation on bacteria. Biotechnology and Bioengineering 15, 707-728.

Champneys, A. R. and Yu. A. Kuznetsov. 1994. Numerical detection and continuation of codimension-two homoclinic bifurcations. Int. J. Bifurcation and Chaos 4, 785-822.

Cunningham, A. and R. M. Nisbet. 1983. Transients and oscillations in continuos culture. In Mathematics in Microbiology, M. Bazin (Ed.), pp. 77-103. London: Academic Press.

De Feo, O. and S. Rinaldi. 1997. Yield and dynamics of tritrophic food chains. The American Naturalist 150, 328-345. 
De Feo, O. and S. Rinaldi. 1998. Singular homoclinic bifurcations in tri-trophic food chains. Mathematical Biosciences in press.

Doedel, E. and J. Kernévez. 1986. AUTO: Software for continuation problems in ordinary differential equations with applications. California Institute of Technology Technical Report, Applied Mathematics, CALTEC, Pasadena.

Drake, J. F. and H. M. Tsuchiya. 1976. Predation on Escherichia coli by Colpoda stenii. Appl. Environ. Microbiol. 31, 870-874.

Ferrière, R., and M. Gatto. 1993. Chaotic population dynamics can result from natural selection. Proceedings of the Royal Society of London B 251, 33-38.

Gilpin, M. E. 1972. Enriched predator-prey systems: Theoretical stability. Science 177, 902-904.

Gragnani, A. and S. Rinaldi. 1995. A universal bifurcation diagram for seasonally perturbed predator-prey models. Bulletin of Mathematical Biology 57, 701712.

Guckenheimer, J. and P. Holmes. 1983. Nonlinear Oscillations, Dynamical Systems and Bifurcations of Vector Fields. New York: Springer Verlag.

Hastings, A. and T. Powell. 1991. Chaos in a three-species food chain. Ecology 72, 896-903.

Hogeweg, P. and B. Hesper. 1978. Interactive instruction on population interaction. Computer, Biology and Medicine 8, 319-327.

Jost, J. L., J. F. Drake, A. G. Fredrickson and H. M. Tsuchiya. 1973. Interactions of Tetrahymena pyriformis, Escherichia coli, Azotobacter vinelandii, and glucose in a minimal medium. Journal of Bacteriology 113, 834-840.

Kauffman, S.A. 1993. Origin of Order: Self Organization and Selection in Evolution. Oxford: Oxford University Press.

Klebanoff, A. and A. Hastings. 1994. Chaos in three-species food chains. Journal of Mathematical Biology 32, 427-451.

Kooi, B. W., M. P. Boer and S. A. Kooijman. 1997, Complex dynamic behaviour of autonomous microbial food chains. Journal of Mathematical Biology in press.

Kot, M., G. S. Sayler and T. W. Schultz. 1992. Complex dynamics in a model microbial system. Bulletin of Mathematical Biology 54, 619-648.

Kuznetsov, Yu. A. 1995. Elements of Applied Bifurcation Theory. New York: Springer Verlag.

Kuznetsov, Yu. A., S. Muratori and S. Rinaldi. 1992. Bifurcations and chaos in a periodic predator-prey model. Int. J. Bifurcation and Chaos 2, 117-128.

Kuznetsov, Yu. A. and S. Rinaldi. 1996. Remarks on food chain dynamics. Mathematical Biosciences 134, 1-33.

May, R. M. 1972. Limit cycles in predator-prey communities. Science 177, 900-902.

McCann, K. and P. Yodzis. 1994. Biological conditions for chaos in a three-species food chain. Ecology 75, 561-564. 
McCann, K. and P. Yodzis. 1995. Bifurcation structure of three-species food chain model. Theoretical Population Biology 48, 93-125.

Muratori, S. and S. Rinaldi. 1992. Low- and high-frequency oscillations in threedimensional food chain systems, SIAM J. Appl. Math. 52, 1688-1706.

Oksanen, L., S. D. Fretwell, J. Arruda and P. Niemela. 1981. Exploitation ecosystems in gradients of primary productivity. The American Naturalist 118, $240-261$.

Pavlou, S. and I. G. Kevrekidis. 1992. Microbial predation in a periodically operated chemostat: a global study of the interaction between natural and externally imposed frequencies. Mathematical Biosciences 108, 1-55.

Rapp, P.E., I.D. Zimmerman, A.M. Albano, G.C. de Guzman and N.N. Greenbaum. 1985. Dynamics of spontaneous neural activity in the simian motor cortex: the dimension of chaotic neurons. Physics Letters 110A, 335-338.

Rinaldi, S., S. Muratori and Yu. A. Kuznetsov. 1993. Multiple attractors, catastrophes and chaos in periodically forced predator-prey communities. Bulletin of Mathematical Biology 55, 15-35.

Rosenzweig, M. L. 1971. Paradox of enrichment: destabilization of exploitation ecosystems in ecological time. Science 171, 385-387.

Sarkar, A. K., D. Mitra, S. Ray and A. B. Roy. 1991. Permanence and oscillatory co-existence of a detritus-based prey-predator model. Ecological Modelling 53, 147-156.

Scheffer, M. 1991. Should we expect strange attractors behind plankton dynamics and if so, should we bother?. Journal of Plankton Research 13, 1291-1305.

Waltman, P. 1983. Competition Models in Population Biology. Philadelphia: Society for Industrial and Applied Mathematics.

West, B.J., and A.L. Goldberger. 1987. Physiology in fractal dimensions. American Scientist 75, 354-365. 